\title{
Brustzentrum am Karl-Olga-Krankenhaus Stuttgart - Modellcharakter für neue Netzwerkstrukturen?
}

\author{
Thomas Kuhn Frank Beldermann
}

Stuttgart hat ein Einzugsgebiet von etwa 1,5 Millionen Menschen, davon erkranken zirka 1000 Frauen pro Jahr an Brustkrebs. Im Jahre 2004 gab es in Stuttgart und naher Umgebung 4 zertifizierte Brustzentren, welche den großen Frauenkliniken angegliedert sind. Nur $50 \mathrm{~km}$ entfernt existiert eines der größten zertifizierten Brustzentren der Umgebung, angegliedert an die Universitätsfrauenklinik Tübingen.

Es klingt daher etwas skurril, dass sich 2004 in Stuttgart 2 Gynäkologen mit senologischer Ausbildung am Karl-OlgaKrankenhaus niedergelassen haben, - der eine ausgebildet an der Universitätsfrauenklinik Heidelberg, der andere am 1. zertifizierten Brustzentrum Deutschlands am Marienhospital in Stuttgart. Die Idee war nicht, «Massen von erkrankten Frauen» zu therapieren, sondern Netzwerkstrukturen zu bilden, die sowohl das Vertrauen der Frauen als auch der zuweisenden Ärzte gewinnen sollten. Die Philosophie des neu gegründeten Brustzentrums an einem Krankenhaus ohne gynäkologische Disziplin war eindeutig: Zwei niedergelassene Senologen übernehmen die Lotsenfunktion in der Diagnostik und Therapie der Brustkrebspatientin. In die Leitungsfunktion des Brustzentrums wurde der kooperierende niedergelassene Radiologe integriert, der ebenfalls in der Klinik vor Ort ist und über alle diagnostischen Interventionstechniken und -verfahren verfügt, einschließlich der präoperativen MRT-gesteuerten Markierung. Onkologisch versierte Kooperationspartner aus dem niedergelassenen Bereich und dem Klinikbereich, - alle am Karl-Olga-Krankenhaus positioniert, ergänzen das Team zu einem interdisziplinären Brustzentrum, entsprechend den senologischen Anforderungen. Die Gemeinschaftspraxis mit dem Schwerpunkt Brustkrebserkrankungen wurde nach den Richtlinien der Dt. Krebsgesellschaft und der Dt. Gesellschaft für Senologie binnen 2 Jahren als Brustzentrum zertifiziert. Das neu gegründete Brustzentrum wollte als fachkompetente Einheit für die erkrankte Frau, aber auch als Dienstleister für zuweisende niedergelassene Frauenärzte und Hausärzte ar- beiten. Aus der Sicht der erkrankten Frau war es oberste Priorität, höchste senologische Kompetenz aus einer Hand zu gewährleisten. Hierbei wurde der Senologe als Lotse etabliert, der die brustkrebserkrankte Frau von der Diagnostik bis zur Therapie begleitet, sozusagen an der Hand nimmt und von Station zu Station geleitet. Somit hat die Patientin stets einen hauptverantwortlichen Ansprechpartner, der Sorge trägt für das Zusammenführen der Befunde bis hin zur Therapieeinleitung. Die gesamte ärztliche Betreuung wird damit ausschließlich durch Fachärzte gewährleistet.

Aus der Sicht der kooperierenden Ärzte musste eine Philosophie erarbeitet werden, die den Dienstleistungsgedanken bis ins kleinste Glied, z.B. den Arzthelferinnen, internalisiert und das virtuelle Brustzentrum als eine Gemeinschaft von Spezialisten nach innen wie nach außen lebt. Der Gynäkologe als Lotse ist dabei nicht hierarchisch den anderen Disziplinen übergeordnet, sondern nimmt eher eine «Primus-inter-paresStellung» ein. Dies ergibt sich vor allem aus der Tatsache, dass die Patientin den Gynäkologen als Hauptansprechpartner ansieht und hiermit gewachsene Strukturen für die Compliance genutzt werden. Diese Compliance wird aber nur dann glaubhaft verstärkt, wenn diese Philosophie ohne Machtgerangel von allen behandelnden Akteuren akzeptiert wird.

Aus der Sicht der zuweisenden Ärzte musste das schon fast evolutionär bedingte ärztliche Konkurrenzdenken durch klare kooperierende Strukturen und abgegrenzte Tätigkeitsfelder des Senologen ersetzt werden. Hierbei ist auch der Dienstleistungscharakter für den zuweisenden Arzt als das oberste Gebot anzusehen, welches sich nicht nur im alltäglichen Umgang, sondern vor allem in der Informationspolitik widerspiegeln muss, auch wenn gerade anfänglich Existenzängste verlocken, die eine oder andere Patientin zu «behalten». Ein klar abgestecktes eigenes Betätigungsfeld sollte die Fachkompetenz des zuweisenden Kollegen ergänzen. Eine solche Kooperation wird auch von den Patientinnen positiv bewertet.

\begin{tabular}{ll}
\hline KARGER & ๑ 2007 S. Karger GmbH, Freiburg \\
Fax +49 7614 520714 & Accessible online at: \\
$\begin{array}{l}\text { E-mail Information@Karger.de } \\
\text { www.karger.com }\end{array}$ & www.karger.com/brc
\end{tabular}

Dr. med. Thomas Kuhn / Dr. med. Frank Beldermann

Zertifiziertes interdisziplinäres Brustzentrum am Karl-Olga-Krankenhaus

Werderstr. 66, 70190 Stuttgart, Germany

Tel. +49 711 2804-020, Fax -0222

E-mail info@Stuttgart-Brustzentrum.de 
Wie aber sieht die praktische Arbeit des interdisziplinären Brustzentrums am Karl-Olga-Krankenhaus in Stuttgart im Alltag aus? Unter einem Dach arbeiten niedergelassene Ärzte der Radiologie, gynäkologischen Onkologie, Pathologie, Psychoonkologie und der internistischen Onkologie. Eigens für das Brustzentrum wurde eine Bruststation etabliert, welche zur Hälfte durch die plastische Chirurgie belegt wird. Für die Brustkrebspatientinnen auf Station sind allein die Gynäkologen Dr. Thomas Kuhn und Dr. Frank Beldermann verantwortlich. Die pflegerische Betreuung auf der Station wird zum Teil durch onkologisch ausgebildete Fachpflegekräfte übernommen. Zuweisende Hausärzte und Frauenärzte überweisen direkt an das Brustzentrum, entweder über den Radiologen oder über die Gynäkologen. Die Abklärung der Befunde findet in gemeinsamer interdisziplinärer Absprache statt. Alle Befunde laufen in kürzester Zeit beim betreuenden Senologen zusammen. Wird ein stanzbioptisch gesichertes Mammakarzinom diagnostiziert, erfolgt das aufklärende Gespräch durch den Gynäkologen, der die Umfelduntersuchungen mit der Patientin koordiniert. Innerhalb von 7 Tagen nach der Biopsie erfolgt das ambulante Staging und die stationäre Einweisung zur operativen Therapie, die in einer interdisziplinären präoperativen Tumorkonferenz festgelegt wurde und die der Patientin vom Senologen erläutert wird. Während des stationären Aufenthaltes von durchschnittlich 4,3 Tagen (die durchschnittliche Aufenthaltsdauer in BadenWürttemberg beträgt 8,1 Tage), erfolgt neben der Wundversorgung die sozialmedizinische Beratung, gegebenenfalls die Versorgung mit einem Prothesen-BH durch das Sanitätshaus, sowie die wichtige Stabilisierung des Arzt-Patienten-Verhältnisses. Arztbriefe über den stationären Aufenthalt und die Therapieempfehlung werden innerhalb von 10 Tagen poststationär an die zuweisenden Ärzte (Radiologe, Hausarzt und Frauenarzt) gesandt. Ist gegebenenfalls eine Chemotherapie notwendig, erfolgt sie durch die gynäkologischen Onkologen im Brustzentrum (also die gynäkologische Gemeinschaftspraxis). Das angegliederte Studienzentrum ermöglicht der Patientin eine enge wissenschaftliche Anbindung. Hierfür sind eigens Studiensprechstunden eingerichtet worden. Portanlage, Echokardiographie, Psychoonkologie bis hin zur Anfertigung einer Perücke werden durch das betreuende Team für die Patientin organisiert. Die obligate Radiatio der operierten Brust übernimmt der Kooperationspartner der Strahlentherapie einer großen Klinik in Stuttgart, welche sich bereits als MVZ (medizinisches Versorgungszentrum) formiert hat. Hierbei sei nochmals unterstrichen, dass der primär aufklärende Gynäkologe bei der Erstdiagnose auch der operierende, der visitierende und schlussendlich auch der Therapie erläuternde Arzt ist. Somit hat die Patientin einen Ansprechpartner während der Therapie ihrer Krankheit. Die nach der Therapie erforderliche Nachsorge erfolgt fast ausschließlich durch die zuweisenden Ärzte.

Sowohl die Anbindung an den onkologischen Schwerpunkt in Stuttgart als auch die eigene strenge Dokumentation der Fälle und die gute Zusammenarbeit mit der Universitätsklinik Tübingen gewährleisten ein Qualitätsmanagement und ermöglichen jederzeit den statistischen Einblick in die eigene Arbeit.

Die Aufgabe der Fort- und Weiterbildung eines Brustzentrums wird durch den angegliederten renomierten Fortbildungsverein GynFo in zahlreichen Veranstaltungen für Kollegen aus der Praxis und Klinik wahrgenommen. Hierbei steht einerseits der Wissenstransfer neuester Therapieempfehlungen im Fokus, aber auch Grundausbildungen in Diagnostik (Mamma-Ultraschall) und die Einführung von Netzwerkstrukturen und Qualitätszirkeln.

Patienteninformationen werden durch das angegliederte Brustforum in Form von Patiententagen oder MammaCareKursen wahrgenommen. Eine eigens eingestellte Ernährungsberaterin, eine Sprechstunde für Komplementärmedizin, eine Sportgruppe für betroffene Frauen und regelmäßig angebotene Tanztherapiekurse komplettieren das Angebot für die Patientin. Hierbei soll der Philosophie Rechnung getragen werden, die Frau als Ganzes im Blick zu behalten.

Als Begründer des interdisziplinären Brustzentrums am KarlOlga-Krankenhaus aus dem niedergelassenen Bereich müssen wir tagtäglich nicht nur den Leitlinien der Dt. Krebsgesellschaft und der Dt. Gesellschaft für Senologie gerecht werden, sondern sind auch den ökonomischen Gesetzmäßigkeiten eines «Unternehmens» unterworfen. So empfinden wir das Gütesiegel der Re-Zertifizierung 2 Jahre nach der Gründung nicht nur als «Lohn» für den täglichen «Kampf» um die im Zentrum stehende Patientin, sondern vor allem als Ermutigung, weiter nach innovativen Versorgungsstrukturen zu suchen. Die hohe Flexibilität in der Reaktion auf sich verändernde Gesundheitsstrukturen, die von den Fachgesellschaften geforderte Qualität und die ökonomischen Anforderungen unseres Gesundheitssystems sind nach unserer Überzeugung nur durch Spezialisierungen in kleinen Einheiten zu erreichen. Hierbei ist der niedergelassene Facharzt genauso qualifiziert, neue Versorgungsstrukturen zu schaffen, wie der Klinikbereich. Der niedergelassene Arzt abstrahiert damit seine Rolle als kooperierender «Großzuweiser» für Kliniken und wird konstruktiver Mitgestalter in der qualitätsgesicherten Versorgung der Brustkrebspatientin! Quod erat demonstrandum! 